\title{
Studying Smartphone Usage: Lessons from a Four-Month Field Study
}

\author{
Ahmad Rahmati, Member, IEEE, and Lin Zhong, Member, IEEE
}

\begin{abstract}
Many emerging mobile applications and services are based on smartphones. We have performed a four-month field study of the adoption and usage of smartphone-based services by 14 novice teenage users. From the field study, we present the application usage and usage characteristics of our participants. We show that their usage is highly mobile, location-dependent, and serves multiple social purposes. Furthermore, we report qualitative lessons regarding the evaluation of smartphone-based services. In particular, we highlight the cases that an accurate evaluation would require a long-term and/or field study instead of a short or labbased study, and the cases where studying a particular application independently is insufficient and a holistic study, i.e., involving the whole device, is necessary. We further present guidelines on effectively shortening the length of a study. These lessons are supported in part by five identified contributing factors to usage evolution.
\end{abstract}

Index Terms-Human-computer interaction $(\mathrm{HCl})$, mobile phones, mobile services, user studies, field studies

\section{INTRODUCTION}

SMARTPHONEs have become a popular platform for mobile Sapplications and services, many of which are evaluated with short-term and often lab-based studies. In this paper, we present findings regarding the usage and usage evolution of mobile devices and services, as derived from both quantitative and qualitative data collected from a fourmonth field study of smartphones provided to 14 teenage mobile users. In particular, we have observed that different usage patterns may apply to different locations for each user, and mobile phones were used considerably when they were indeed close to PCs that were accessible to users.

Furthermore, we present qualitative lessons learned regarding the evaluation of services based on smartphones, In particular, we show that it is often crucial to have longterm studies in real-life settings and examine the device and its coexisting services in a holistic manner to accurately evaluate the usability of mobile devices and services. We identify five factors that contribute to the usage evolution and user-perceived usability of a mobile service. The five factors include the initial opinion users hold toward a mobile service, the required knowledge and skill to operate the service, the context dependence of its usage, the natural process of boring the user, and the process that the user personalizes his/her device. We further show that it may take many weeks to converge.

Throughout the reported field study, we have gathered both qualitative and quantitative data from focus groups, interviews, and in-device logging. The field study was carried out from late 2007 to early 2008 with 14 teenagers from Pecan Park, Houston, TX, an underserved community in a major urban area in the USA. Our participants had little

- A. Rahmati is with Broadcom Corporation, Sunnyvale, CA.

E-mail:ahmad@rahmati.com.

- L. Zhong is with Rice University, Houston, TX. E-mail: lzhong@rice.edu. Manuscript received 16 Feb. 2010; revised 26 Oct. 2011; accepted 27 Apr. 2012; published online 31 May 2012.

For information on obtaining reprints of this article, please send e-mail to: tmc@computer.org, and reference IEEECS Log Number TMC-2010-02-0077. Digital Object Identifier no. 10.1109/TMC.2012.127. or no prior experience with smartphones, providing a unique opportunity to study the adoption and usage evolution of smartphone-based services. Importantly, we do not claim that the services available on the smartphones used in our study are representative of all mobile services, especially newer ones. Yet we do believe that usage evolution is intrinsic and the five factors identified in our study will provide key insights into the evaluation of many, if not most, mobile services. To optimize the instrumentation software and formulate initial hypotheses, we conducted a one-month pilot study before the long-term study. The same experimental smartphones were used in the pilot study, but with 10 students from Rice University, all majoring in engineering. Findings from the study were reported in [1].

The remainder of the paper is organized as follows: We discuss related work in Section 2. We present an overview of the participants of our user study and their community in Section 3 and explain our research methods in Section 4. We present the findings of our user study regarding the application usage and usage characteristics of our participants in Section 5. We present our findings regarding the usage evolution of the phones, including the five identified contributing factors, their supporting cases, and lessons learned in Section 6, and conclude in Section 9.

\section{Related Work}

Usability of mobile phones and services has been the subject of intensive research. Kjeldskov and Graham [2] have reviewed research methods of 102 Mobile human-computer interaction (HCI) publications, of which 42 focus on evaluation. Of the 42 , only 19 percent employ field studies, while 71 percent employ lab-based experiments. Kjeldskov and Graham conjectured that lab-based studies limit the development of knowledge on mobile HCI.

Our work further supports their argument. Recent work has also suggested the importance of long-term studies in real-life context settings for the evaluation of mobile devices 
and services For example, Kangas and Kinnunen [3] stress the importance of usage context, but stop at providing the user as much context that "buttons and software that feels like it is actually working." Sarker and Wells [4] find that characteristics of the user, phone, task, mobility, and context all affect usage. Blom et al. [5] acknowledge that understanding the users context and even culture is necessary for assessment of a mobile phone, and presents their experience with several methods of obtaining usage information in real-life context. Roto et al. [6] use cameras mounted on the phone to assess device usage in naturalistic settings. Kaikkonen et al. [7] find that while task execution times are similar between laboratory and field settings, observed usability problems were significantly different. These works and others [8], [9], [10], [11], [12], [13] highlight the importance of performing user studies in real-life settings, outside of lab environments.

There have been several studies of mobile phone usage, after the one we report in this paper. For example, Falaki et al. study the application, network, and energy use patterns of 33 Android users, and the application use patterns of an additional 200 Windows Mobile users [14], and show the high diversity of users. Bohmer et al. study the application installation and use patterns of 4,000 anonymous Android users during four months [15], and show the correlation of application use with time and prior application use. Do et al. look into logs from 77 Symbian users over nine months, and also show that mobile usage is location dependent [16]. Similarly, our recent work collects detailed usage and context information from 34 iPhone 3GS users over one year [17], [18]. Compared to them, this paper studies the users and their usage patterns in a more holistic way, with significantly more qualitative data regarding the users, and through focus groups and interviews, even though this study is more limited in in-device logging. More importantly, this paper reports on a user study that was conducted in late 2007, just before the widespread adoption of smartphones, in part fueled by the iPhone and Android platforms. Its findings serve as a valuable snapshot of usability and adoption at that time, in particular since none of the participants had smartphones prior. Therefore, this paper can serve as a reference point for the evolution of smartphone usage.

Many existing work address a specific aspect of mobile phone design, such as the user interface (e.g., input methods [19] and navigation [20]), availability [21], acquisition and replacement [22], personalization [23], or Internet connectivity [24]; or specific applications and services, such as text messaging [25], [26], [27], mobile web [28], the integrated camera [29], [30], text entry [6], and mobile video [31], [32]. Social aspects of mobile phone usage have also been widely studied [33], [34], [35], [36], [37], [38], for example, social significance [33] and the characteristics of mobile communication [34] and application usage [38]. In contrast, our focus is on the holistic usage of the mobile phone and its services for ICT access.

Our findings not only confirm the importance of longterm holistic studies in natural settings, but present lessons for the evaluation of smartphone-based mobile services. In particular, we show the circumstances in which an accurate evaluation would require long term and/or holistic field studies. Further, we provide insight into how study length, context, and usage of coexisting services impact the usage and evaluation of a specific service.

\section{Community and Participants}

Our study took place in Pecan Park, an underserved community in Houston, Texas, where the average household income is below the poverty line. Approximately 13 percent of US residents are below the poverty line, which is $\$ 10,400$ for a single person family, and increases $\$ 3,600$ per additional person [39]. Researchers from Rice University and Technology for All (TFA), a local nonprofit organization, have installed an open-access 802.11 network covering a significant portion of the community including residential areas, public schools, and parks (tfa.rice.edu).

\subsection{Long-Term Study Participants}

We were able to recruit 14 teenage participants from Pecan Park for the long-term study. They were between 15 and 18 years old, either attending or had just finished high school. The participants had little or no prior experience with smartphones, providing a relatively clean slate for studying the usage evolution of smartphonebased services. In the remainder of this paper, we use "participants" and "primary group" to refer to them, unless otherwise indicated.

All participants had PC-based Internet access at school and a good command of Internet knowledge. They used Internet-based research for their homework, using Wikipedia and Google. They were also familiar with and used social network sites, in particular MySpace. All but one had access to PCs at home; seven had PCs devoted to them. PC ownership and regular Internet access set this underserved community, i.e., an urban one in a developed country, apart from those in developing countries.

Four of our participants had their own GSM phone plans, and used their SIM cards in our smartphones. For them, we provided $\$ 20$ gift cards at each focus group meeting as compensation. We gave the other participants prepaid SIM cards and provided \$25 refill cards at each focus group, equivalent to between 130 to 150 minutes. We provided several tutorial sessions to participants on how to operate the experimental phone and its various features at the beginning of the study. We also provided technical support to all participants throughout the study to ensure a smooth experience.

We believe the smartphones, plans, and gift cards provided reasonable monetary, educational, and recreational incentive for participation, and we were well known among the community. However, it was especially difficult to recruit willing participants. While we liked, and would have benefitted from more detailed logging, it would have made it more difficult, or potentially impossible to recruit a reasonable number of participants.

\subsection{Control Group Participants}

During the course of the long-term study, we formulated new research hypotheses for which we had not collected proper data in earlier focus groups. For example, we hypothesized that a change of specific behavior and assessment was related to an improvement in typing skills, 


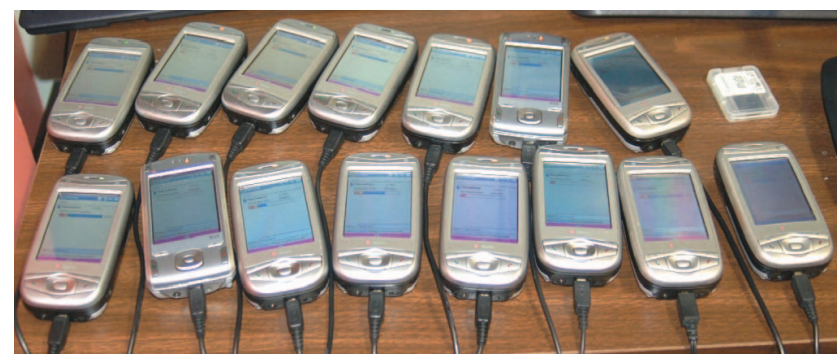

Fig. 1. Experimental mobile phones were tested in the lab before the study.

but we had not assessed the participants' typing skill at the beginning of the study.

To compensate, we recruited an additional 10 participants from the same community for a single 80 minute user study session. They were in the same age range and had similar ICT experience as those in the primary group, and we assume the results from the control group would be similar to what would have been attainable from the longterm group at the beginning of the study. In each session, several participants were shown the phone, and asked about their opinions about various aspects of the smartphones. We also tested their typing speed. We provided each participant with a $\$ 20$ gift card.

\section{Research Methods}

\subsection{Experimental Phone}

We prepared an experimental HTC Wizard phone for each participant by developing and installing Visual $\mathrm{C}++$ based logging software that runs in the background (see Fig. 1). The logging software reduces the standby battery lifetime of the smartphones from about five to three days. The participants were informed of the battery lifetime from the start. At the beginning of the long-term study (Summer 2007), the Wizard, branded as T-Mobile MDA and Cingular 8125, was one of the most feature-rich commercial Pocket PC smartphones. It is a Bluetooth and Wi-Fi capable GSM phone with a 2.8-inch QVGA touch-screen display. It has a sliding hardware QWERTY keyboard in addition to a small on-screen keyboard for use with a stylus, and handwriting recognition. We supplied a 1-GB MiniSD storage card and USB cable with the smartphones. The phone takes under 1 minute to boot; however, the user is not required to boot the phone unless it crashes or runs out of battery. Under normal usage, the phone will go in a standby mode when not used and can resume operation virtually instantaneously, at a push of a button. Table 1 provides a list of some of its applications and features.

Due to the inherent privacy concern with underage participants (under 18 years old), we did not log actual application usage. Instead, we relied on qualitative data for such information. Our logging software records the battery level, charging status, and display status (on/off) every minute. It also records visible $\mathrm{Wi}-\mathrm{Fi}$ access points and their signal strength every 5 minutes. The logging frequencies were determined based on our previous study [1]. We identify interactive (i.e., nonphone) sessions lasting more than 2 minutes using the display status. The phone display
TABLE 1

Applications Available on the Experimental Phones

\begin{tabular}{|l|l|}
\hline \multirow{2}{*}{ Communication } & $\begin{array}{l}\text { Text Messaging (SMS), Instant Mess- } \\
\text { aging, Email (Outlook / Web based) }\end{array}$ \\
\hline \multirow{2}{*}{ Recreational } & Media Player, Games, Camera \\
\cline { 2 - 2 } Work / Educational & $\begin{array}{l}\text { Internet Explorer (IE) } \\
\text { Acrobat }\end{array}$ \\
\cline { 2 - 2 } $\begin{array}{l}\text { Personal Information } \\
\text { Management (PIM) }\end{array}$ & Address Book, Calendar, Task List \\
\hline
\end{tabular}

turns off after 1 minute of phone conversation or nonusage. Therefore, we assume the device is being used interactively when the display is continuously on for 2 minutes or more. Wi-Fi information can be used to directly calculate approximate location [40], [41]. However, we did not attempt to do so due to privacy considerations. Instead, we employed the Wi-Fi traces to cluster the most visited access points into areas according to their proximity. Each cluster corresponds to a unique physical area, enabling us to study the location dependence of phone usage with minimal disclosure of location information. Our location clustering method is limited to locations with visible $\mathrm{Wi}$-Fi access points. Most of our participants indeed spent a significant portion of their lives in such locations; the average among all participants was 73 percent. To deal with locations without visible Wi-Fi access points, we cluster them together as a single area.

\subsection{Qualitative Data Collection}

We held two focus group meetings every three weeks; each participant could choose to attend either one of the two. Each focus group took about 70 minutes and took place at the conference room of a nonprofit organization in the community with two research team members attending. The focus groups were semistructured. Before each focus group, we prepared the topics and questions based on results from our previous study and the analysis of existing data, in particular recently collected data. We occasionally interviewed a participant if there were issues particular to him or her. The focus group conversations were recorded with the consent of the participants, transcribed, and used alongside our notes for manual coding. In addition to using the coded data, we often revisited the audio files for context in the later analysis of the coded data.

\section{Phone Usage}

Using the quantitative and qualitative data from our longterm study participants over four months, in this section we report on what applications were used, and how the phones were used.

\subsection{Applications}

\subsubsection{Recreational}

We found that recreational applications, such as media player, games, and to a lesser extent the camera were the most popular type of applications on the phones. All of our participants mentioned the use of at least one recreational 
application in each focus group. They started using the built-in games immediately, and quickly learned to load MP3 files on the MiniSD card. By the end of the first month, most participants had music collections on the phone. Our participants reported that they primarily used these applications in their free times and often used them socially and shared them with their peers, as will be further addressed in Section 5.2.

Media player remained popular throughout the study, and one participant even sold his iPod, since he "always had the phone with him." On the other hand, although most participants had found and installed new games, gaming popularity dropped toward the end of the study and they often complained that the available games were boring.

We hypothesize that the attraction of recreational applications is positively correlated with their freshness; therefore, phones should allow and simplify the process of refreshing refresh recreational applications and/or content. Our experimental phones have very limited gaming ability and the available games can lose their freshness rather quickly. On the other hand, new games were costly to our participants and required advanced technical knowledge for installation. As a result, gaming gradually lost its attraction. We note that Windows Mobile phones at the time did not have a built-in application store, and users were required to manually find, download, and install applications. Also, social networking games, for example, on Facebook, were unavailable at the time on our phones and may have different dynamics. In contrast with games, our participants found it easy to obtain music in standard MP3 or WMA formats and load them to the phones. Therefore, media player had sustained its freshness through new music content and, therefore, remained popular throughout our study. Findings from our more recent study using iPhones [17], [18] confirm this hypothesis; while individual games quickly drop in usage, users regularly install new games from the Apple App Store.

\subsubsection{Internet and Communication}

While the experimental phones are capable of GPRS/EDGE, our participants did not have cellular data plans during the study. Instead, they had to use available Wi-Fi services for Internet connectivity, including the community open Wi-Fi network and their school Wi-Fi network. There are two technical shortcomings for Wi-Fi to provide ubiquitous wireless connectivity, in comparison with cellular data services. First, the community Wi-Fi network is intended for outdoor coverage and only one participant had usable signal inside the home. Second, Wi-Fi provides inadequate support for mobility. As a result, our participants reported disconnections when moving around outdoors. These two shortcomings presented a severe usability challenge, for example, for instant messaging (IM), which will be detailed later in Section 6.

Although our participants did not have a cellular data plan, all of them told us they would like data access and they were willing to pay for ubiquitous Internet access if the plan were cheaper; they mentioned acceptable and affordable prices as between $\$ 1$ to $\$ 10$ per month. At the time, cellular data plans typically cost $\$ 20$ to $\$ 30$ per month.

Our experimental phones provided email and IM, in addition to voice communication and text messaging (SMS).
During the training sessions, we showed the participants how to create an email address for those who did not have one already, and how to retrieve and send them on the phone using the included Outlook software and otherwise. However, our participants never used email for personal communication, and only occasionally used it for work related communications.

On the other hand, online social networking had become extremely popular among our target population, and all of our participants had MySpace accounts. We must note that the heavy MySpace pages were poorly supported by the phones. Our participants also reported that they regularly used IM to communicate with their friends when using a PC. Initially, they were eager to use IM on the experimental phone. However, their enthusiasm disappeared a few weeks into the study due to the wireless connectivity problems mentioned above. We discuss this in further detail in Section 6.

Our participants extensively used text messaging. Furthermore, most of them reported an increase in their amount of text messaging, indicated by changing their plan to one with an increased or unlimited number of included text messages, or by using more of their prepaid minutes for texting.

\subsubsection{Work/Educational}

While not as popular as recreational applications, many of our participants used the phone for productivity applications and web surfing, often to fulfill their duties, such as schoolwork. By the second month of the study, they had used word mobile to write their homework. They used email to send their homework, and used Internet explorer to research their material. On the other hand, they did not report any use of Acrobat, Excel, or PowerPoint on the phones. According to their self reports, their usage was based on location and context, for example, when they were in bed, or when they did not have access to PCs. For example, one participant reported that they use word mobile to finish late homework at school. Another one used the phone for schoolwork when he was hesitant to use a family PC due to a quarrel.

\subsection{Characteristics}

The small form factors and long battery lifetime make mobile phones highly accessible as they can be taken to any location and be used immediately, even on the go. Such high accessibility enables their nonvoice applications to be highly mobile and used in situations where PCs may not be used. We next present findings regarding this unique feature of mobile phones and on location-based usage, from both qualitative and quantitative data.

\subsubsection{Portable Usage}

Due to the overhead of operating portable PCs, i.e., space requirement, startup time, and short battery lifetime, the usage of PCs is at most portable, instead of truly mobile. In contrast, we would expect significant usage of phones in all areas, even those which participants spend little time at.

Our logging software records visible Wi-Fi access points and as described in Section 4.1, we have used the traces to cluster the most visited access points into unique physical areas while maintaining user privacy. Figs. $2 a$ and $2 b$ show 


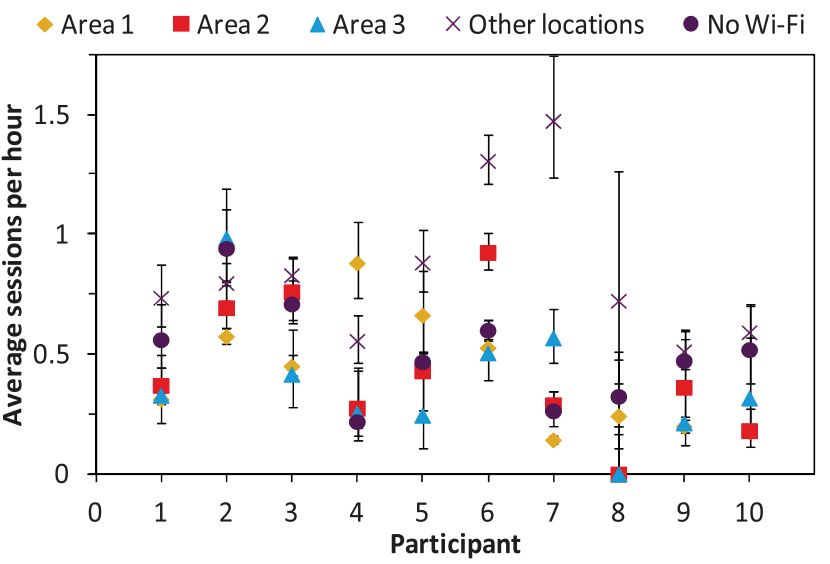

(a) Number of sessions (average $=0.42$ / hour)

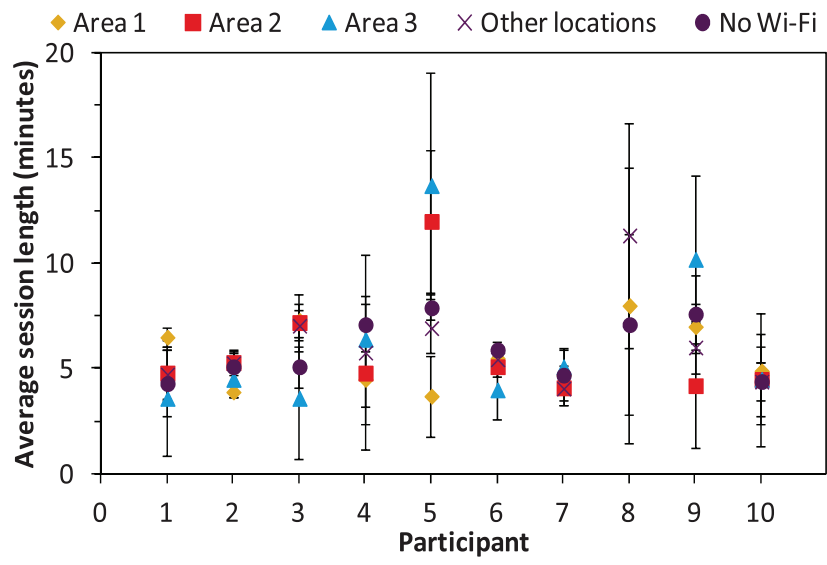

(b) Length of sessions (average $=6.0$ minutes)

Fig. 2. Usage patterns of our participants. Areas 1 to 3 denote the top three location clusters where each participant spent their time, and were calculated for each participant separately. Locations that could not be classified due to lack of visible Wi-Fi access points are shown collectively as "No Wi-Fi." Whiskers indicate 95 percent confidence intervals.

the average number of nonvoice sessions per hour and the average session length at each user's top three location areas, respectively, for 10 participants who we collected sufficient data.

This quantitative evidence shows that indeed, most participants extensively used nonvoice phone applications on-the-go and at locations where they spent a relatively small portion of their time. These locations are aggregated and shown as "other locations" in Fig. 2. On average, our participants spent 16 percent of their time in all these locations, with less than 6 percent in their fourth location area, and less than 2 percent in each other location area. Most of our participants used nonvoice phone applications at a significantly higher frequency at locations which they spend little time (see Fig. 2a). Our participants had an average 0.84 sessions per hour and 6.1 minutes per session at these locations, compared to a total average of 0.42 sessions per hour and 6.0 minutes per session.

Our qualitative data provides macroscopic fine-grained location information regarding usage. Our participants regularly mentioned how the high mobility of the phone can enable truly mobile usage at a microscopic level (e.g., at different locations inside home), which is beyond the reach of portable PCs. As another example, some participants leveraged the mobility of phones to facilitate Internet connectivity; they would go to specific locations in their homes for a better Wi-Fi signal. One of them sometimes even walked two blocks to a neighborhood park to use the free Wi-Fi network with the phone. Indeed, while a significant part of the community is covered by the community Wi-Fi, there are many dead spots, in particular indoors.

\subsubsection{Phones Used at Home, Alongside PCs}

While our location areas are calculated anonymously for each participant, we can safely assume the area where the phones spend most of their time (Area 1) is their home. Often, this includes the time when participants are asleep. We can see that the phones are used extensively at Area 1, presumably home, as well, where many participants have access to a PC. Indeed, for all participants, the average number of sessions per hour in Area 1, and their average lengths, are comparable with their participants' other areas, i.e., well within the same order of magnitude. This indicates that This indeed corroborates with qualitative evidence from our focus groups.

Furthermore, as shown in Fig. 3, there is no significant difference in the ratio of phone usage at home versus everywhere between participants with devoted personal PCs and those without (Participants 3, 5, 8, and 9). This indicates that the mobile phones indeed provide unique values at home in comparison with PCs. Otherwise, better access to PCs would have led to reduced use of mobile phones.

\subsubsection{Discreet Usage}

The small portability, small form factor, and accessibility of mobile phones make it possible for users to access ICT in a discreet and private manner. Discreet usage refers to use under social context that conspicuous ICT access is considered inappropriate, disallowed, or simply uncomfortable.

The most prominent case is that phone usage was generally disallowed in the high schools our participants attend, except during lunch breaks. Based on the data log as reported earlier and the focus group discussion, it is obvious that this rule was routinely circumvented. We also have numerous self-reported incidents in addition to the
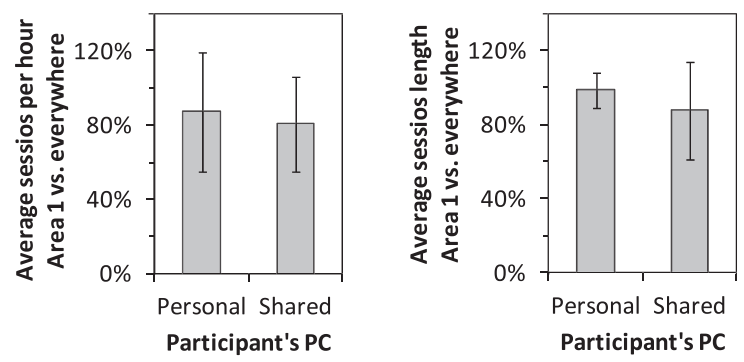

Fig. 3. Phones provide unique values at home in comparison with PCs. Otherwise, better access to PCs would have led to reduced use of mobile phones. Proportion of usage at Area 1 (presumably home) versus everywhere, for participants with devoted access to a personal PC, versus participants with shared access to a PC. Whiskers indicate 95 percent confidence intervals. 
statistics of phone usage indicating a large number of application sessions took place during school hours.

After getting the experimental phone, our participants quickly recognized and learned discreet uses. Participants in the very first focus group almost unanimously agreed that the experimental phone is difficult to hide because it is large and unlike nonsmartphones, requires two-handed operation, for example, when texting. By the second focus group, instead of complaints about size, we got stories explaining how they hide the device during usage, for example, in the classroom, which would be impossible to carry out on laptops. The discreet usage includes rushing homework with the built-in word mobile, checking emails, text messaging, and gaming. One participant even told us how he used the phone in a way similar to a piece of paper to exchange messages back and forth within the classroom.

It is important to note that while such discreet usage may help users achieve their short-term objectives, they may be detrimental to their best interest, in particular when they are minors. It is also challenging to control discreet usage while respecting user privacy.

\subsubsection{Social Motivations}

The high accessibility of mobile phones allows them to be carried and used in public as well as privately, serving social purposes for their users. Previous studies have already shown mobile phones function as social symbols and fashion accessories [33]. Our experimental phones allow personalization similar to PCs in addition to ringtones and user interface options found on regular phones. Our participants leveraged this and extensively personalized the appearance and functionality of the phones; they reported use in public places and social situations so that the personalization was visible or audible. The personalization was such that from the mid of the study, we were able to tell which participant was the user of a phone just based on the phone appearance.

In addition to personalization, the participants used the phones' features for social purposes as well. For example, the experimental phones have Wi-Fi capability that was uncommon even for Smartphones. One of the participants noted that the Wi-Fi capability was recognized and admired by some of her peers who own Smartphones due to the high speed of Wi-Fi compared to her peers' data plans.

\subsubsection{Sharing}

An interesting finding from this study is that our participants extensively shared their experimental phones with others to achieve their social objectives. We believe mobile phones are better suited to sharing than PCs due to two reasons. First, their high accessibility brings more sharing opportunities with lower overhead, as sharing is contextual. Second, the perceived risk for phone sharing is smaller than PC sharing: sharing bicycle is less difficult than sharing a car. This is due to fewer recognized risky usage patterns, simpler functionalities, and more straightforward restoration through resetting. Most of our participants with personal PCs told us they were wary of sharing PCs mentioning reasons ranging from fear of viruses and malware to deleting important files.
TABLE 2

Popular Applications Change during the Study

\begin{tabular}{|c|c|c|c|c|}
\hline Beginning & & lidway & \multicolumn{2}{|c|}{ Towards the end } \\
\hline$\leftarrow$ & \multicolumn{2}{|c|}{ Text Messaging } & & $\rightarrow$ \\
\hline \multicolumn{5}{|c|}{$\leftarrow$ Instant Messaging $\rightarrow$} \\
\hline & $\leftarrow$ & \multicolumn{2}{|c|}{ Word Mobile } & $\rightarrow$ \\
\hline$\leftarrow$ & Games & $\rightarrow$ & & \\
\hline$\leftarrow$ & \multicolumn{3}{|c|}{ Media Player } & $\rightarrow$ \\
\hline$\leftarrow$ & \multicolumn{3}{|c|}{ IE } & $\rightarrow$ \\
\hline \multicolumn{5}{|c|}{$\leftarrow$ Stylus text entry $\rightarrow$} \\
\hline & $\leftarrow$ & \multicolumn{2}{|c|}{ Hardware keyboard text entry } & $\rightarrow$ \\
\hline
\end{tabular}

Based on our findings, we hypothesize that a user makes a decision regarding phone sharing based on perceived gain, in personal prestige and social capital, and perceived risk, in privacy and security, which are two opposite forces. While we observed that sharing is an important way for mobile phones to provide social values to our participants, existing phones provide inadequate support for sharing with privacy assurance. When a mobile user shares their phone, they essentially give away complete access to the phone applications and data. While it may seem tempting to simply apply typical PC access control to phones, for example adding a "guest" account, it cannot support the dynamic policies that allow the owner to grant different temporary users with access to different services and data in situ. For example, one may want to share some photos with a family member while sharing a song with a classmate. To effectively assist sharing, the access control must support intuitive ways to specify policies, for example, designed for one main user and multiple temporary users, and being able to quickly specify what services and data to share with a temporary user. This has motivated the design of xShare, as reported in [42].

\section{UsAge Evolution}

Our participants' usage changed considerably over the course of the study. Table 2 summarizes the applications and features that were popular at different stages of the study, as were reported in our focus groups. While many factors can affect usage, we are interested in how users explore various aspects of the smartphones and embrace them into their lives. Through this process, users assess the usability and usefulness of a feature and their usage changes as their assessment converges. In this section, we first present quantitative evidence of usage change, and then present five contributing factors to usage evolution.

Using the logged data, we found quantitative evidence for change in usage amount. Fig. 4 presents weekly statistics for nonvoice phone usage by all participants in three measures: average length of usage sessions, average usage time per hour, and average number of sessions per hour. Fig. 4 presents strong evidence of usage change over time. First, we can see that phone usage is significantly higher in the first week, in all three measures. This suggests that the initial excitement about the phone led to increased usage. Second, we can see that it took up to five to six weeks for 


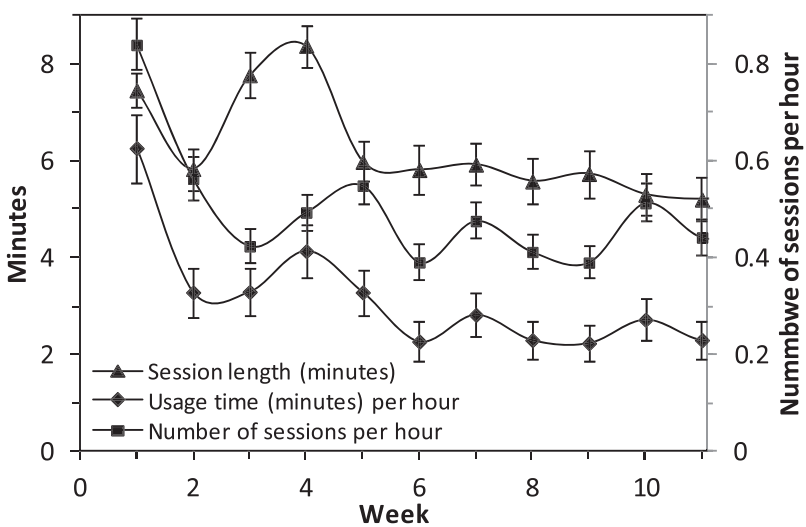

Fig. 4. Usage drops quickly, takes five to six weeks to stabilize. Whiskers indicate 95 percent confidence intervals.

our participants' usage to stabilize. This shows the necessity of long studies to correctly assess the usability and values of smartphones. We consider the convergence of usage amount as an indicator for the lower bound of a valid study length.

\subsection{Contributing Factors to Usage Evolution}

Our study shows that the assessment and usage variation can take a long time to converge. Based on our observations, we have identified five qualitative, impactful contributing factors that we can employ to help interpret usage evolution. We call these contributing factors to usage evolution. It is important to note that we do not claim that these are all possible contributing factors. Further studies may indeed find even more factors.

- Initial opinion. The user's subjective assessment of devices and services is affected by the initial opinion, bias, and first impression they hold regarding the functionality or prestige associated with the device or service. It takes time for their opinion to change and converge on the final assessment.

- Knowledge and skills. Higher knowledge and skill requirement for using a service lead to longer assessment times because the user learns to effectively use the service. In contrast to initial opinion, knowledge and skills can be objectively measured.

- Context dependence. Features useful or frustrating under more limited context require longer time because the user has to be in the context to experience the values and problems.

- Boredom. The attraction of applications can drop as their novelty wears out with prolonged use. In contrast with initial opinion, boredom is not caused by an unrealistic initial opinion, but is the natural, long-term loss of interest in an application. Boredom especially applies to recreational and entertainment applications.

- Personalization. Personalization of a new device takes time, and can significantly affect the usage of the device.

\subsection{Supporting Cases}

As supporting evidence, we provide example cases for each of the contributing factors for usage evolution.

\subsubsection{Initial Opinion}

Text entry is an excellent example of the effects of initial opinion, demonstrated by the attitudes of participants in our long-term study and those in our control group. In the training sessions, we had presented both stylus-based text entry and the hardware keyboard. At the beginning, our long-term study participants regularly used stylus-based text entry (on-screen keyboard and handwriting recognition) over the hardware keyboard. Similarly, after we demonstrated the three input methods, our control group participants initially preferred stylus-based over hardware keyboard-based text entry. Both groups demonstrated an initial opinion toward stylus-based text entry, mentioning their perception regarding its ease of use and its novelty and coolness. Furthermore, we noticed a different bias in the long-term study participants by contrasting their attitude changes with those of our control group. All but one control group participants changed their opinion and preferred the hardware keyboard over the stylus-based text entry after they tried all three methods in the focus group, typing in a sentence. However, it took our long-term participants more than a month to really embrace the hardware keyboard, citing its accuracy. We attribute the difference to an additional bias our long-term participants may have had: the prestige gained from using stylus-based text entry before peers, who described it with comments such as "it's cool." Our control group only tried the experimental phones in a single user study session, where every participant was given one to play with. They, therefore, were less likely subject to the prestige and peer opinion-based bias.

Change in the type of ownership prestige was another example of initially biased reaction. Our findings indicate there are two distinct types of prestige related to mobile phone usage: the first is for the possession of expensive objects and the second for the access to valued functions. Indeed, the high accessibility of mobile phones allows them to be carried and used in public as well as privately, serving social purposes for their users. Previous studies have already shown mobile phones function as social symbols and fashion accessories [33].

Initially, the first type of prestige, for the possession of the expensive phone, was dominant. In the first two rounds of focus groups, we got a large number of comments highlighting the perceived value of the phones among our participants' peers. For example, one participant told us "[my friends] would say "that's a cool phone"... they really like it but it's kind of expensive." However, we have found that the initial opinion toward prestige due to the expense of the object was later overshadowed by the prestige brought by having access to the functionality provided by the phones. Such prestige is even more apparent when the phone carries a unique function, for example, Wi-Fi. The shift in ownership prestige was indicated by the transition from demonstrating the phones to sharing them with their peers, and more importantly the responses highlighting the value of certain functionalities of the phone. For example, one participant told us "my friends still like it, because of Windows Media Player it's partly an MP3 player." Another noted that the Wi-Fi capability was recognized and admired 

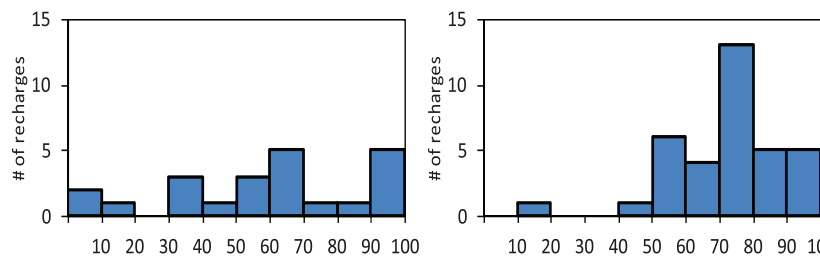

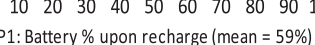

$\begin{array}{llllllllll}10 & 20 & 30 & 40 & 50 & 60 & 70 & 80 & 90 & 100\end{array}$

P1: Battery \% upon recharge $($ mean $=73 \%)$

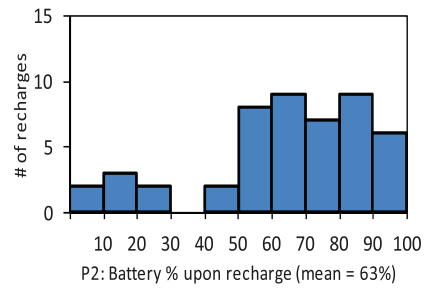

(a) First month of study

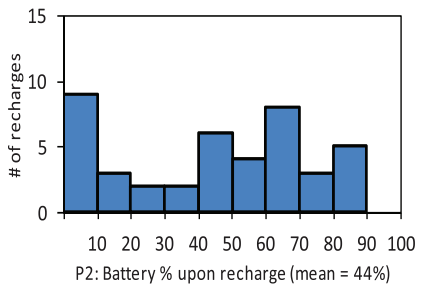

(b) Third month of study

Fig. 5. Participants take over a month to adapt to battery life.

by some of her peers who own Smartphones due to the high speed of Wi-Fi compared to her peers' data plans. Furthermore, the transition from demonstrating the phone to sharing it represents a shift in prestige, from conspicuous consumption to an interest in gaining social capital.

\subsubsection{Knowledge and Skills}

The charging pattern of our participants is a clear example of the time required to acquire knowledge regarding certain features. As mentioned in Section 4.1, our logging software automatically logs battery levels and charging status. ${ }^{1}$ The remaining battery levels upon recharge for two indicative participants, extracted from our logs are shown in Fig. 5. The qualitative reports let us interpret the changes. Three weeks into the trial, P1 told us that she had found the battery to last three days, and she had to charge it on the second half of the third day at most. Later on, she told us that she now checks the battery percentage level in the extended battery information screen of the phone and charges before it reaches 50 percent. The logs confirm that she rarely went below 50 percent in the third month. Another participant, P2 regularly charged the phone during the first month. In the third month of the study, he told us how the phone battery lasts a second day without charging. Our logs confirm that he charged at reduced battery levels during the third month, on average, at 44 percent, from 63 percent in the first month. From the battery level and charging status logs and self-reported data from our participants, we can see that some participants take one to two months to learn and adapt to the battery lifetime of their phone.

Our participants' assessment and use of word mobile is a clear example of the time required to acquire skills for certain applications. At the beginning of our study, participants reported they do not find word mobile useful and rarely use it. Similarly, our control group participants dismissed word mobile in the focus group meetings. However, toward the mid of the long-term study, our long-term participants started using word mobile to write

1. We have reported findings regarding the charging patterns in [43]. However, the data is used here to support a different case.

homework when they had no access to a PC. We believe this change in assessment and usage was related to their improved typing skills. Unfortunately, we had not quantitatively assessed their typing speed at the beginning of the study. Therefore, we used our control group as an approximate of the long-term group at the beginning of the study. The control group's assessment of Word was similar to the initial assessment of the long-term participants. We used the same procedure to measure the typing speed of both groups on the hardware keyboard of the phones and on a PC keyboard. While the typing speeds of the two groups were similar on the PC keyboard, they were significantly different on the phone keyboards. On the phones, our field study participants typed at 18 to 40 words per minute (WPM), with an average of 28 , at the end of the study. In contrast, our control group typed at 14 to 24 WPM, with an average of 18. Such significant difference in their typing speed explains their different assessments of Word, which has a high text entry requirement.

\subsubsection{Context Dependence}

IM was a significant example of the context-dependence factor in addition to initially biased reaction. Our participants were avid IM users on PCs. From the beginning of the study, they made serious attempts to use the IM software on the phones and aggressively sought our assistance when there was a problem in the first three weeks. Although we resolved the problem, the popularity of IM died very soon, and most participants told us they had used it "only a few times" afterward, and they prefer to use text messaging because the IM software "gets disconnected" when they move around, due to limited Wi-Fi coverage and Wi-Fi's inherent lack of support for mobility. Indeed, over time, the novelty of IM communication with their peers had wore off and they had encountered contextual situations where IM had become frustrating to use.

Location dependence of nonvoice application usage is another example of context dependence. Our logging software records visible $\mathrm{Wi}-\mathrm{Fi}$ access points and as described in Section 4.1, we have used the traces to cluster the most visited access points into unique physical areas while maintaining user privacy. Figs. $2 \mathrm{a}$ and $2 \mathrm{~b}$ show the average number of nonvoice sessions per hour and the average session length at each location, respectively, for 10 participants who we collected sufficient data. It shows that most of our users have significantly different usage characteristics at different locations, in terms of average session lengths and/or sessions per hour, hence their usage was location dependent.

\subsubsection{Boredom}

Our observations regarding recreational applications are a clear indication of boredom affecting the usage of the device. Note that recreational applications, such as media player, games, and to a lesser extent the camera were the most popular type of applications on the phones. Media player remained popular throughout the study, and one participant even sold his iPod, since he "always had the phone with him." On the other hand, gaming popularity dropped toward the end of the study and the participants often complained that the available games were limited and 
boring, and new games are hard to find and install, and sometimes costly. Indeed, our participants found it easy to obtain music in standard (e.g., MP3) formats and load them to the phones. On the other hand, our experimental phones have very limited gaming ability and the available games can lose their freshness rather quickly. As a result, gaming gradually lost its attraction.

\subsubsection{Personalization}

Phone sharing among our participants was a clear example of personal data stored on the phones affecting their usage. Personal data impacts sharing as it can increase the perceived risk of sharing. From the very beginning, our participants actively started demonstrating the phones with their peers and family. Soon afterward, participants started sharing the phones with their peers. However, as the participants used the experimental phones, they gradually stored an increasing amount of personal content and unintentional traces in the phone Examples include text messages, photographs, call history, and browsing history. Privacy concern became widespread by the second and third focus group meetings, and most participants had become more sensitive about their personal data in the phone.

Our participants changed their usage patterns by reducing their sharing circle to more trusted friends and relatives and/or by taking more precautions. For example, one participant told us how he has to delete each text message two times (from his inbox and deleted items folders) to prevent others from accessing them when he shared the phone. Many participants asked for better privacy protection and access control for the wide range of private data on their phone during sharing, which is indeed unavailable on existing phones. We must note that the perceived social gain or loss of sharing or not sharing the device may be influenced by environmental conditions. For example, peer pressure can increase the perceived social loss of not sharing the device. In one such example, a participant told us she was worried about her personal data, but was pressured into sharing her phone with others, due to being physically small.

\subsection{Lessons Learned}

To leverage the identified contributing factors in the evaluation of mobile devices and services, the researcher must first determine which of the contributing factors would influence the evaluation results. We answer the following important questions regarding the evaluation of mobile services based on our study.

\subsubsection{Is a Lab Study Sufficient, or is a Field Study Necessary?}

Our study shows that, if the evaluation is expected to be influenced by the initial opinion, context dependence, boredom, or personalization factors, a field study is necessary. However, if the evaluation is mainly affected by the knowledge and skills factor, a lab study is sufficient. Indeed, among the identified contributing factors, a lab study can only measure the knowledge and skills factor accurately. However, as we showed in Section 6.2.1, a lab study may be influenced by a different set of initial opinions than real-life usage in the field. Further, a lab study cannot provide real-life context or personalization, nor will it last long enough for the effects of boredom to be realized.

\subsubsection{Is a Holistic Study Necessary?}

Our study highlights that in many cases, the adoption, usage, and value of services must not be studied independently, but in a holistic system-wide level, i.e., allowing and considering the use the phone and its services as a whole. Note that a field study may or may not be holistic. A holistic study is necessary when the evaluation is expected to be influenced by knowledge and skills that can be acquired from other applications, or when the evaluation is expected to be influenced by the sharing of the phone, which is in turn affected by the personalization factor. The rationale is as follows.

First, the knowledge and skills factor implicates that two applications will affect each other's usage evolution if they require a common skill, which we call correlated training. As noted previously, our long-term participants took quite some time to develop a positive assessment of word mobile, and text messaging, which was extensively used throughout the study, may have contributed significantly to the participants' typing speed and comfortableness with word mobile.

Second, personal data stored on the phone created by a certain application can make users reluctant to sharing their phone as a whole, impacting other applications that may have no privacy implications, for example, media player or games. In our study, text messages were the most sensitive personal data reported by the participants. While the text messaging application was rarely if ever shared, participants' sensitivity toward text messages reduced their willingness to share their phones in whole, impacting other applications.

\subsubsection{What Is the Minimum Length of a Field Study?}

A study must be long enough to allow each of the contributing factors relevant to an evaluation to stabilize. For example, if personal data and the personalization of the phone can affect usage, the study must allow enough time for participants to personalize the phone and load it with personal data. On the other hand, a simple skill may be acquired in a relatively short study session.

The study must also be long enough to allow the overall device usage amount to stabilize. This is clearly a lower bound of a study length. In our case, the usage logs indicated that while the number of usage sessions per hour converges in two to three weeks, it took approximately five to six weeks for the overall device usage to converge (see Fig. 4). Clearly, individual applications may need more time, especially if they are dependent on specific skills or personalization, or subject to the effects of boredom. In such cases, the change in the usage amount of the particular application may be too small to be apparent in the device usage logs. We conjecture that a per-application usage log may be a more effective indicator. However, it would also increase privacy concerns that may in turn affect adoption and usage.

\subsubsection{How Can a Researcher Shorten a Field Study without Jeopardizing Its Validity?}

Given the context dependence of usage evolution, as shown in Section 6.2.3, it may seem tempting to select participants 
who more often encounter context relevant to the feature being studied, or even artificially subject participants to relative context. While this can help usage to converge faster, the biased context may indirectly affect the accuracy of results due to the effects of correlated training (defined in Section 7.3.2). The reason is that the selected users may be less exposed to contexts promoting applications that help develop skills useful for the subject application. In our case, had we selected users mainly based on interest or context pertaining to word mobile, they may have never obtained the typing skills necessary for Word during the course of our study if they were not been interested in other more simple applications that improve typing skills, for example, text messaging.

On the other hand, according to the contributing factors, facilitating personalization of the device and the learning of knowledge and skills can help accelerate usage evolution. There are many methods to achieve this, some examples are as follows: First, selecting participants who already use the same phone as the study, or one close to it can effectively shorten the time required for personalization and acquiring knowledge and skills in regard to the phone. Second, if the users do not have prior experience with the phones, preloading them with personal data and recreational content or holding introductory sessions focusing on such items can accelerate the personalization of a device. Third, training sessions can be employed to expedite skills and knowledge acquisition. Finally, selecting participants with existing social connections, and/or promoting interaction between participants, for example, focus groups and social events, can help participants learn from each other. Indeed, we had many examples throughout our study where one participant figured out something new and this knowledge was propagated to other participants in either the focus groups or at school.

We also note that it is possible to promote adoption and reduce convergence time by considering the contributing factors when designing the phone and applications, i.e., by facilitating knowledge/skill acquisition, taking advantage of initial opinion, and simplifying personalization. For example, a feature that requires an advanced skill may be facilitated by an attractive, simple game that requires the same skill but at a lower level, similar to our observation that text messaging may have improved the text entry speed and eventually promoted the adoption of word mobile.

\section{Conclusion}

Our four-month field study of the usage and usage evolution of smartphone-based services shows that our participants used the devices in a highly mobile fashion even within the same area, and at different locations. We observe that different usage patterns may apply to different locations for each user, and smartphones were used considerably even when users were close to PCs that were accessible to them. We show that our participants creatively leverage the high accessibility of the phones not only for ICT access but for social purposes as well.

Our study goes beyond presenting the usage and adoption of smartphones, and provides lessons learned regarding the evaluation of mobile services and devices.
These lessons are supported in part by five identified contributing factors that can be employed to interpret usage evolution: initial opinion, knowledge and skills, context dependence, boredom, and personalization. We show the circumstances in which an accurate evaluation would require long term, holistic, and/or field studies: A holistic study is necessary when the evaluation results would be influenced by personalization affecting the sharing of the phone, or by knowledge and skills. A field study is necessary when the evaluation results are affected by factors other than knowledge and skills. A study must be long enough to allow not just the device usage to stabilize, but allow all contributing factors relevant to the study to stabilize. Finally, we show that while a biased context may shorten the study length, it may jeopardize its results. On the other hand, a study can be effectively shortened by facilitating personalization and knowledge/skill acquisition during the study.

\section{ACKNOWLEDGMENTS}

The authors would like to thank Bryan Grandy and Jim Forrest from Technology for All (TFA) for their help with the user studies. This work was supported in part by US National Science Foundation Awards IIS/HCC \#0803556, NeTS-WN 0721894, and CRI/IAD 0751173.

\section{REFERENCES}

[1] A. Rahmati and L. Zhong, "Usability Evaluation of a Commercial Pocket PC Phone: A Pilot Study," Proc. ACM Int'l Conf. Mobile Technology, Applications and Systems (Mobility), 2007.

[2] J. Kjeldskov and C. Graham, "A Review of Mobile HCI Research Methods," Proc. ACM Int'l Conf. Human Computer Interaction with Mobile Devices and Services (MobileHCI), 2003.

[3] E. Kangas and T. Kinnunen, "Applying User-Centered Design to Mobile Application Development," Comm. ACM, vol. 48, pp. 5559, 2005.

[4] S. Sarker and J. Wells, "Understanding Mobile Handheld Device use and Adoption," Comm. ACM, vol. 46, pp. 35-40, 2003.

[5] J. Blom, J. Chipchase, and J. Lehikoinen, "Contextual and Cultural Challenges for user Mobility Research," Comm. ACM, vol. 48, pp. 37-41, 2005.

[6] V. Roto, A. Oulasvirta, T. Haikarainen, J. Kuorelahti, H. Lehmuskallio, and T. Nyyssonen, "Examining Mobile Phone Use in the Wild with Quasi-Experimentation," technical report, Helsinky Inst. of Information Technology, 2004.

[7] A. Kaikkonen, A. Kekäläinen, M. Cankar, T. Kallio, and A. Kankainen, "Usability Testing of Mobile Applications: A Comparison between Laboratory and Field Testing," J. Usability Studies, vol. 1, no. 1, pp. 4-17, Nov. 2005.

[8] T. Kallio and A. Kaikkonen, "Usability Testing of Mobile Applications: A Comparison between Laboratory and Field Testing," J. Usability Studies, vol. 1, pp. 4-16, 2005.

[9] R. Schusteritsch, C. Wei, and M. LaRosa, "Towards the Perfect Infrastructure for Usability Testing on Mobile Devices," Proc. SIGCHI Conf. Human Factors in Computing Systems (CHI), 2007.

[10] P. Hagen, T. Robertson, M. Kan, and K. Sadler, "Emerging Research Methods for Understanding Mobile Technology Use," Proc. Australia CHISIG Conf. Computer-Human Interaction, 2005.

[11] J. Donner, "Research Approaches to Mobile Use in the Developing World: A Review of the Literature," The Information Soc., vol. 24, pp. 140-159, 2008.

[12] A. Dix, Human-Computer Interaction. Pearson/Prentice-Hall, 2004.

[13] J. Froehlich, M.Y. Chen, S. Consolvo, B. Harrison, and J.A. Landay, "MyExperience: A System for in Situtracing and Capturing of User Feedback on Mobile Phones," Proc. ACM MobiSys, 2007.

[14] H. Falaki, R. Mahajan, S. Kandula, D. Lymberopoulos, R. Govindan, and D. Estrin, "Diversity in Smartphone Usage," Proc. ACM MobiSys, 2010. 
[15] M. Bohmer, B. Hecht, J. Schoning, A. Kruger, and G. Bauer, "Falling Asleep with Angry Birds, Facebook and Kindle: A Large Scale Study on Mobile Application Usage," Proc. ACM Int'l Conf. Human Computer Interaction with Mobile Devices and Services (MobileHCI), 2011.

[16] T.M.T. Do, J. Blom, and D. Gatica-Perez, "Smartphone Usage in the Wild: A Large-Scale Analysis of Applications and Context," Proc. 13th Int'l Conf. Multimodal Interfaces (ICMI), 2011.

[17] C. Shepard, A. Rahmati, C. Tossell, L. Zhong, and P. Kortum, "LiveLab: Measuring Wireless Networks and Smartphone Users in the Field," ACM SIGMETRICS Performance Evaluation Rev., vol. 38, pp. 15-20, 2010.

[18] A. Rahmati, C. Tossell, C. Shepard, P. Kortum, and L. Zhong, "Exploring iPhone Usage: The Influence of Socioeconomic Differences on Smartphone Adoption, Usage and Usability," Proc. ACM Int'l Conf. Human Computer Interaction with Mobile Devices and Services (MobileHCI), 2012.

[19] A.K. Karlson and B.B. Bederson, "One-Handed Touchscreen Input for Legacy Applications," Proc. SIGCHI Conf. Human Factors in Computing Systems (CHI), 2008.

[20] M. Ziefle, U. Schroeder, J. Strenk, and T. Michel, "How Younger and Older Adults Master the Usage of Hyperlinks in Small Screen Devices," Proc. SIGCHI Conf. Human Factors in Computing Systems (CHI), 2007.

[21] K. Sadler, T. Robertson, and M. Kan, "'It's Always There, It's Always On': Australian Freelancer's Management of Availability Using Mobile Technologies," Proc. ACM Int'l Conf. Human Computer Interaction with Mobile Devices and Services (MobileHCI), 2006.

[22] E.M. Huang and K.N. Truong, "Breaking the Disposable Technology Paradigm: Opportunities for Sustainable Interaction Design for Mobile Phones," Proc. SIGCHI Conf. Human Factors in Computing Systems (CHI), 2008.

[23] S.Y. Ho and S.H. Kwok, "The Attraction of Personalized Service for Users in Mobile Commerce: An Empirical Study," ACM SIGECOM Exchange, vol. 3, pp. 10-18, 2003.

[24] H. Verkasalo, "Analysis of Mobile Internet Usage among EarlyAdopters," Info - J. Policy, Regulation, and Strategy for Telecommunications, vol. 11, pp. 68-82, 2009.

[25] R.E. Grinter and L. Palen, "Instant Messaging in Teen Life," Proc. ACM Conf. Computer Supported Cooperative Work, 2002.

[26] R. Grinter and M. Eldridge, "Y do Tngrs Luv 2 Txt Msg?" Proc European Conf. Computer Supported Cooperative Work, 2001.

[27] A. Battestini, V. Setlur, and T. Sohn, "A Large Scale Study of TextMessaging Use," Proc. ACM Int'l Conf. Human Computer Interaction with Mobile Devices and Services (MobileHCI), 2010.

[28] Y. Cui and V. Roto, "How People Use the Web on Mobile Devices," Proc. Int'l Conf. World Wide Web (WWW), 2008.

[29] D. Okabe, J. Chipchase, M. Ito, and A. Shimizu, "The Social Uses of Purikura: Photographing, Modding, Archiving, and Sharing," Proc. PICS Workshop, UbiComp, 2006.

[30] D. Okabe, "Emergent Social Practices, Situations and Relations through Everyday Camera Phone Use," Proc. Conf. Mobile Comm. and Social Change, 2004.

[31] Y. Cui, J. Chipchase, and Y. Jung, "Personal TV: A Qualitative Study of Mobile TV Users," Proc. Fifth European Conf. Interactive TV: A Shared Experience, 2007.

[32] K. O'Hara, A.S. Mitchell, and A. Vorbau, "Consuming Video on Mobile Devices," Proc. SIGCHI Conf. Human Factors in Computing Systems (CHI), 2007.

[33] L. Srivastava, "Mobile Phones and the Evolution of Social Behaviour," Behaviour and Information Technology, vol. 24, p. 111, 2005.

[34] M. Ito and O. Daisuke, "Mobile Phones, Japanese Youth, and the Re-Placement of Social Contact," Proc. Ann. Meeting Soc. for the Social Studies of Science, 2001.

[35] A.S. Taylor and R. Harper, "Age-Old Practices in the 'New World': A Study of Gift-Giving between Teenage Mobile Phone Users," Proc. SIGCHI Conf. Human Factors in Computing Systems (CHI), 2002.

[36] S. Berg, A.S. Taylor, and R. Harper, "Mobile Phones for the Next Generation: Device Designs for Teenagers," Proc. SIGCHI Conf. Human Factors in Computing Systems (CHI), 2003.

[37] M. Ito and D. Okabe, "Technosocial Situations: Emergent Structurings of Mobile Email Use," Personal, Portable, Pedestrian: Mobile Phones in Japanese Life, M. Ito, D, Okabe, and M. Matsuda, eds., MIT Press, 2005.
[38] H. Verkasalo and H. Hämmäinen, "A Handset-Based Platform for Measuring Mobile Service Usage," Info - J. Policy, Regulation, and Strategy for Telecommunications, vol. 9, pp. 80-96, 2007.

[39] "Annual Update of the HHS Poverty Guidelines," Dept. of Health and Human Services, Office of the Secretary, Fed. Register, Jan. 2008.

[40] A. LaMarca, Y. Chawathe, S. Consolvo, J. Hightower, I. Smith, J. Scott, T. Sohn, J. Howard, J. Hughes, and F. Potter, "Place Lab: Device Positioning Using Radio Beacons in the Wild," Proc. Int'l Conf. Pervasive Computing (Pervasive), 2005.

[41] Y.C. Cheng, Y. Chawathe, A. LaMarca, and J. Krumm, "Accuracy Characterization for Metropolitan-Scale Wi-Fi Localization," Proc. ACM MobiSys, 2005.

[42] Y. Liu, A. Rahmati, Y. Huang, H. Jang, L. Zhong, Y. Zhang, and S. Zhang, "xShare: Supporting Impromptu Sharing of Mobile Phones," Proc. ACM MobiSys, 2009.

[43] A. Rahmati and L. Zhong, "Human-Battery Interaction on Mobile Phones," Pervasive and Mobile Computing, vol. 5, pp. 465-477, 2009.

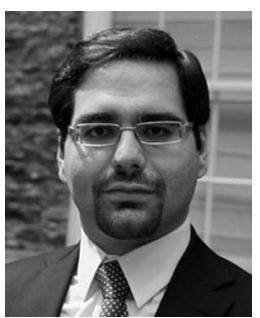

Ahmad Rahmati received the BS degree in computer engineering from the Sharif University of Technology in 2004 and the MS and PhD degrees from the Department of Electrical and Computer Engineering, Rice University, in 2008 and 2012. He is currently a senior staff scientist in the Mobile and Wireless Group at Broadcom. His publications received the ACM MobileHCl Best Paper Award in 2007 and were featured twice as the spotlight paper of the IEEE Transactions on Mobile Computing, in 2010 and 2011. His research interests include mobile and wireless system design and applications, contextaware computing through sensing and statistical learning on large data, as well as human factors and $\mathrm{HCl}$. He is a member of the IEEE.

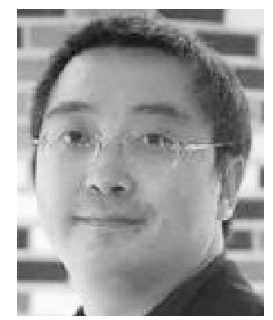

Lin Zhong received the BS and MS degrees from Tsinghua University in 1998 and 2000, respectively, and the $\mathrm{PhD}$ degree from Princeton University in September 2005. He is currently an associate professor in the Department of Electrical and Computer Engineering, Rice University. He is a recipient of the US National Science Foundation CAREER award and best paper awards from ACM MobiSys 2011, IEEE PerCom 2009, and ACM MobileHCl 2007. A paper he coauthored was identified as one of the 30 most influential papers in the first 10 years of the Design, Automation and Test in Europe conference. His research interests include mobile computing, human-computer interaction, and nanoelectronics. He is a member of the IEEE.

For more information on this or any other computing topic, please visit our Digital Library at www.computer.org/publications/dlib. 